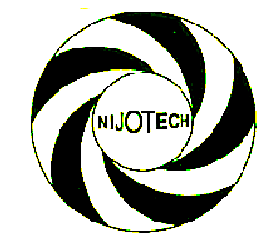

Nigerian Journal of Technology (NIJOTECH)

Vol. 33. No. 1, January 2014, pp 104- 113

Copyright(C) Faculty of Engineering,

University of Nigeria, Nsukka, ISSN: $1115-8443$

www.nijotech.com

http://dx.doi.org/10.4314/njt.v33i1.14

\title{
PERFORMANCE SIMULATION OF A NATURAL CIRCULATION SOLAR AIR HEATER WITH PHASE CHANGE MATERIAL ENERGY STORAGE
}

\author{
A. I. Obi ${ }^{1,}{ }^{*}$, A. O. Odukwe' ${ }^{2}$, S. O. Enibe $^{3}$ \\ 1 Department of Mechanical EngineERIng, Madonna University, AKPugo Campus, Nigeria \\ 2,3 Department of Mechanical EngineERING, UNIVERSITY OF Nigeria, NSUKKA, NigERIA \\ E-mailaddresses.1amarachukwu.obi@unn.edu.ng,2okay.odukwe@yahoo.com, \\ 3samuel.enibe@unn.edu.ng
}

\begin{abstract}
The one-dimensional radial heat conduction in a phase change material (PCM) heat storage system that is encapsulated in cylindrical pipes in a single glazed flat plate natural circulation solar air heater is presented. The PCM is prepared in modules, with the modules equispaced across the absorber plate. Enthalpy method is applied to convert the relevant energy balance equations into dimensionless forms for easy tracking of the moving phase boundaries. Crank-Nicolson implicit finite difference scheme which has the features of being stable, accurate and fast in its solution is used in the solution of the governing equations subject to suitable initial and boundary conditions. The scheme is applied at each node and the resulting simultaneous equations are solved using the Gauss-Seidel iterative method. An existing computer programme in BASIC known as the EGGINC which was developed for the rectangular channel containing the PCM is modified to cylindrical coordinate for the pipes containing the PCM to predict the temperature distributions in the solar air heater. The predicted temperatures of the system are compared with the experimental data under daytime no-load condition over the ambient temperature range of 18.5$36.0^{\circ} \mathrm{C}$ and daily global irradiation of $4.9-20.1 \mathrm{MJ} / \mathrm{m}^{2}$-day. The predicted temperatures were found to agree closely with experimental data.
\end{abstract}

Keywords: Phase change material; Natural circulation; Solar air heater; implicit finite difference; Predicted temperatures

\section{Introduction}

The heat-conduction equation and its solutions express temperature as a function of the space coordinate and time. This work uses an existing numerical technique - the Crank-Nicolson implicit finite difference scheme for solving onedimensional radial problem of a tube containing phase change material in a natural circulation solar air heater. In particular we will consider the melting/freezing problem using the enthalpy method, hence, the temperature range in the phase change using the heat conduction equation. Crank-Nicolson method provides a good introduction in the phase change problem and presents an elaborate approximation for these problems. The implicit methods are the natural alternative to the front tracking methods. Within these implicit methods the most widely used are the enthalpy method. In the enthalpy method described in [1], the total enthalpy of the system, is utilized.
In many industrial problems the phase change occurs over a temperature range rather than at a specified temperature. The enthalpy method can also be applied to such problems [2]. These methods appear to have great flexibility and are easily extended to multidimensional problems. The advantages of this approach are stated in [1]. The solar air heater is considered to be a promising direction for increasing the economic feasibility of low temperature solar systems for heating water for domestic, agricultural and industrial applications. A system of this type combines collection and storage of thermal energy in a single unit [3]. Most investigators have devoted greater attention to forced circulation air heaters operating under steady state conditions. In contrast, only few reports of natural circulation air heaters have appeared in the literature [4].

In this work, cylindrical tubes or pipes encapsulating phase change materials (wax) are

* Corresponding author, Tel: +234-8037326685 
used instead of rectangular blocks or channels as reported in [2]. In the previous work the analysis was on rectangular coordinates whereas in this work, herein, cylindrical coordinate is used extensively in the analysis. This work was validated and optimized using an upgraded computer simulation program, based on an optimally verified transient thermal analysis. Natural circulation air heaters are important in many industrial and agricultural applications including the drying of crops and medicinal/aromatic plants, timber, natural rubber, tea and coffee products, and fodder for animals [5], [6], [7] and [8]. It could also be used for poultry egg incubation. In this case, as well as in the drying of medicinal/aromatic plants, the heated air temperatures was maintained within specified ranges. Further, the hot air is to be supplied over a continuous period of several days, including off-sunshine periods. For these special applications, some form of energy storage, possibly combined with an auxiliary heat source is required. Other applications of the natural circulation solar air heaters are in domestic hot water and space heating, and to a lesser degree, in industrial processes. They can be employed to supply hot water in absorption refrigeration systems for space cooling [13].

\section{Objective of Study}

- To obtain a long time approximate solution for the temperature distribution in a solar air heating system with pipes containing phase change material in cylindrical coordinate.

- To modify PCM module of the existing computer program to account for the phase change material in cylindrical coordinates.

- To test the modified programme with experimental data.

- Finally to optimize the performance of the solar air heater

\section{Scope of Study}

The bulk of this work centres on writing a computer programme to find the temperature history $\theta(r, t)$ inside an infinitely long cylinder of radius $r$ that is initially at a uniform temperature $\theta_{0}$ and it contains wax which melts as the temperature rises at a temperature of $\theta_{\mathrm{i}}$. The thermal diffusivity and conductivity of the material of the cylinder are $\alpha$ and k, respectively, [10] and [2].

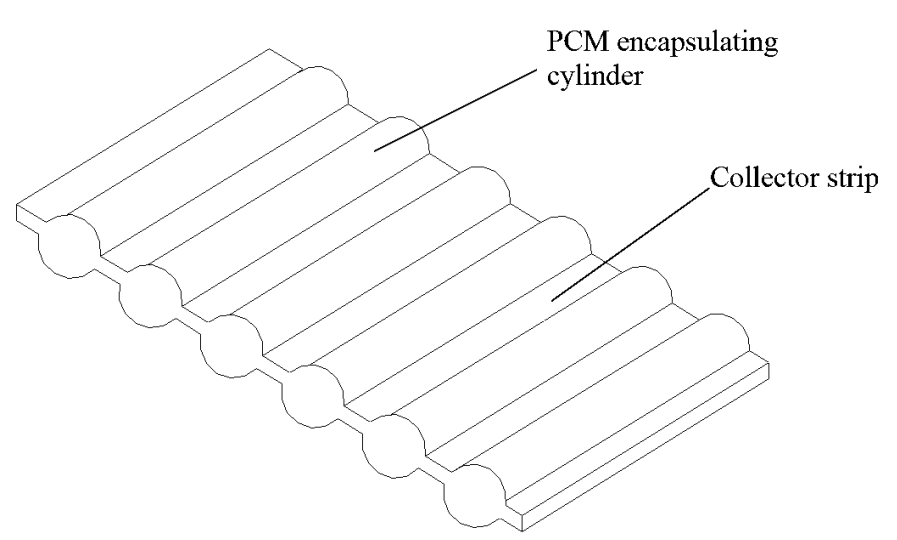

Figure 1: The collector plate geometry.

\section{Derivation of the Simultaneous Equations for the Temperature Distributions in a Solar Air Heater}

The 1-D differential equation governing heat transfer in cylindrical coordinates is given by

$\rho_{s} c_{s} \frac{\partial T_{s}}{\partial t}=k_{s}\left\{\frac{1}{r} \frac{\partial T_{s}}{\partial r}+\frac{\partial^{2} T_{s}}{\partial r^{2}}\right\}$

We assume that the heat flows in the $\mathrm{y}$ and $\mathrm{z}$ directions are negligible. The initial condition is

$T_{s}(r, 0)=f(r), 0 \leq r \leq r_{m}$

Where $f(r)=T_{s}=T_{\text {ambient }}$

The boundary conditions are as follows:

(i) At the edge of the pipe, the radius $r=r_{m}$ is maximum and there is continuation of heat flux.

$\therefore-k_{s} \frac{\partial T_{s}}{\partial r}=U_{s}\left(T_{p 1}-T_{s}\right)$

(ii) At the centre of the pipe, the radius is $r=$ 0 and there is temperature symmetry.

$\therefore \frac{\partial T_{s}}{\partial r}=0$

To overcome the problems of tracking the moving boundary associated with the melting/freezing of the phase change material, we employ the total enthalpy method described in [1] and [2]. Here, the heat transfer equation is rewritten in terms of total enthalpy of the phase change material as

$\rho_{s} \frac{\partial H_{s}}{\partial t}=\frac{1}{r} \frac{\partial T_{s}}{\partial r}+\frac{\partial^{2} T_{s}}{\partial r^{2}}$

The phase change material used does not melt at a single temperature, but rather over a temperature range say $\mathrm{T}_{1}$ and $\mathrm{T}_{2}$. The temperature - enthalpy relationships for the material may be represented as a series of straight lines as shown in table 1 and Figure 2. 
Table 1: Temperature-Enthalpy Relationship For Paraffin Wax (PCM)

\begin{tabular}{cc}
\hline Temperature $(\mathrm{K})$ & Specific Enthalpy $\left(\mathrm{kJ} \mathrm{kg}^{-1}\right)$ \\
\hline 273 & $0=\mathrm{H}_{0}$ \\
331 & $52.2=\mathrm{H}_{1}$ \\
333 & $268.4=\mathrm{H}_{2}$ \\
373 & $304.4=\mathrm{H}_{3}$ \\
\hline
\end{tabular}

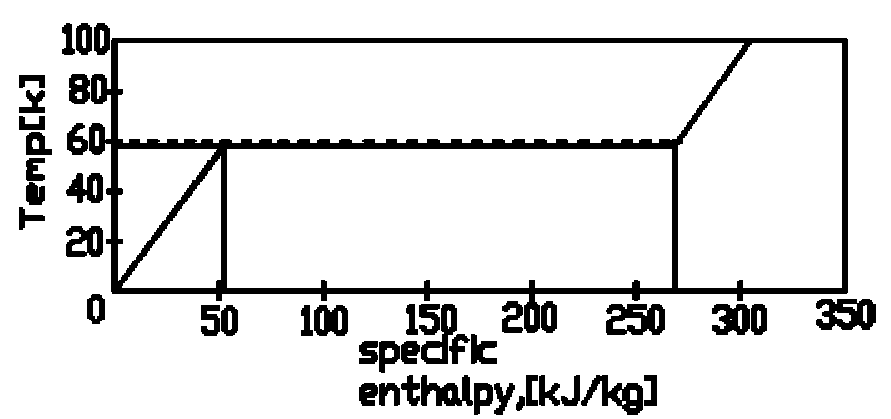

Figure 2: Temperature-Enthalpy Relationship for Paraffin Wax(PCM)

Suppose the specific enthalpy of the PCM at a reference temperature $T_{0}$ is $H_{0}$, the specific enthalpy of the fully solid phase at a minimum melting temperature $\mathrm{T}_{1}$ is $\mathrm{H}_{1}$, while the specific enthalpy of the liquid phase at the maximum melting temperature $\mathrm{T}_{2}$ is $\mathrm{H}_{2}$. The specific enthalpies are calculated from the expressions presented in [2] as follows:

$H_{1}=c_{s}\left(T_{1}-T_{0}\right)$

$H_{2}=H_{1}+c_{s}\left(T_{2}-T_{1}\right)+\Delta H_{s}^{\prime}=H_{1}+\Delta H_{s}$

where $\mathrm{T}_{0}$, is an arbitrary reference temperature, and $\Delta H_{s}=\Delta H_{s}^{\prime}+c_{s}\left(T_{2}-T_{1}\right)$, the modified enthalpy of fusion. We assume that at $\mathrm{T}_{1}$, the PCM is fully solid, while at $\mathrm{T}_{2}$, it is fully liquid. In between the two temperatures, the liquid fraction and the temperature vary linearly with specific enthalpy.

For any temperature less than $T_{1}$, the specific enthalpy is given by the expression $H_{s}=H_{0}+c_{s}\left(T_{s}-T_{0}\right), \quad T_{s}<T_{1}$

Suppose the liquid fraction at any point between the minimum and maximum melting temperature is $\alpha$. Then, the specific enthalpy of a mixture of the liquid and solid phase at any temperature, $\mathrm{T}_{\mathrm{s}}$ is given by

$$
H_{s}=H_{1}+\alpha \Delta H_{s}
$$

$T_{s}=T_{1}+\alpha \Delta T_{s}=T_{1}+\alpha\left(T_{2}-T_{1}\right), T_{1} \leq T_{s} \leq T_{2}$
Above the maximum melting temperature $\mathrm{T}_{2}$, the PCM is fully liquid and the specific enthalpy is given by

$$
H_{s}=H_{2}+c_{s}\left(T_{s}-T_{2}\right), T_{s}>T_{2}
$$

Since $\mathrm{T}_{0}$ can be taken quite arbitrary, we set $\mathrm{H}_{0}=$ 0 at $\mathrm{T}_{0}=273 \mathrm{k}$.

To simplify the utilization of the temperatureenthalpy relationships in the governing equations, we define the dimensionless enthalpy, $\Psi_{s}$ as

$$
\begin{aligned}
& \Psi_{s}=\frac{H_{s}-H_{0}}{\Delta H_{s}}=\frac{H_{s}}{\Delta H_{s}} \\
& \text { Let } \Psi_{1}=\frac{H_{1}}{\Delta H_{s}}
\end{aligned}
$$

and

$$
\Psi_{2}=\frac{H_{2}}{\Delta H_{s}}
$$

For the range $\mathrm{T}_{\mathrm{s}}<\mathrm{T}_{1}$, and from equations (8) and (12) we have

$$
\therefore T_{s}=\frac{\psi_{s} \Delta H_{s}}{c_{s}}+T_{0}
$$

For the range $T_{1} \leq T_{s} \leq T_{2}$ we have from equation (9) that

$H_{s}=H_{1}+\alpha \Delta H_{s}, \quad T_{1} \leq T_{s} \leq T_{2}$

But $\alpha=\frac{T_{s}-T_{1}}{T_{2}-T_{1}}=\frac{T_{s}-T_{1}}{2 \epsilon_{s}}$

Hence, $H_{s}=H_{1}+\frac{T_{s}-T_{1}}{2 \epsilon_{s}} \Delta H_{s}$

Dividing through by $\Delta H_{s}$, we have

$$
\begin{aligned}
& \Psi_{s}=\Psi_{1}+\frac{T_{s}-T_{1}}{2 \epsilon_{s}} \\
& \Rightarrow 2 \epsilon_{s}\left(\Psi_{s}-\Psi_{1}\right)=T_{s}-T_{1}
\end{aligned}
$$

For the range $\mathrm{T}_{\mathrm{s}}>\mathrm{T}_{2}$, we have from equation (11) that

$$
H_{s}=H_{2}+c_{s}\left(T_{s}-T_{2}\right), \quad T_{s}>T_{2}
$$

Since $H_{s}=H_{0}+\Psi_{s} \Delta H_{s}$

$$
T_{s}=\frac{\Delta H_{s}}{c_{s}}\left(\psi_{s}-\psi_{2}\right)+T_{2}
$$

where $\mathrm{H}_{0}$ is set equal to zero, and $H_{2}=\Psi_{2} \Delta H_{s}$, we obtain

From equations (15), (19) and (20) we can write in general that

$$
T=\lambda_{1} \psi+\lambda_{2}
$$

Where $\lambda_{1}, \lambda_{2}$ are constants which depends on the temperature range. 
From equation (21), we can write more specifically for the phase change material as

$T_{s}=\lambda_{1 s} \psi_{s}+\lambda_{2 s}$

where it is understood that $\lambda_{1 \mathrm{~s}}$ and $\lambda_{2 \mathrm{~s}}$ are evaluated at $\mathrm{T}_{\mathrm{s}}$. From equation (21) and equation (22), the difference between the temperature of any material and that of the phase change material, $\mathrm{T}-\mathrm{T}_{\mathrm{S}}$ may be given by:

$T-T_{s}=\lambda_{1} \psi+\lambda_{2}-\lambda_{1 s} \psi_{s}-\lambda_{2 s}$

$T-T_{s}=\lambda_{2}\left[\psi+\left(\lambda_{2}-\lambda_{1 s} \psi_{s}-\lambda_{2 s}\right) / \lambda_{1}\right]$

$\therefore T-T_{s}=\lambda_{1}\left(\psi-\psi_{s}^{*}\right)$

where $\psi_{s}^{*}=\left(\psi_{s} \lambda_{1 s}+\lambda_{2 s}-\lambda_{2}\right) / \lambda_{1}$

On the other hand, for any two materials not involving the phase charge material such as $\mathrm{T}_{\mathrm{f} 1}$ and $\mathrm{T}_{\mathrm{f} 2}, \lambda_{1}$ and $\lambda_{2}$ are constants and equal, hence, the temperature difference is given by

$T_{f 1}-T_{f 2}=\lambda_{1}\left(\psi_{f 1}-\psi_{f 2}\right)$

To simplify the numerical solution of the equation and also aid in the parametric analysis of the natural circulation, solar air heater, we define the following dimensionless parameters.

$$
\begin{aligned}
R & =\frac{r}{r_{m}} \\
\tau & =\frac{t}{t_{o}}
\end{aligned}
$$

where $t_{0}$ is some arbitrary time interval

Utilizing the above dimensionless enthalpy terms, the differential coefficients may be given as follows:

$$
\begin{aligned}
& \frac{\partial H_{s}}{\partial t}=\frac{\Delta H_{s}}{t_{0}} \frac{\partial \psi_{s}}{\partial \tau} \\
& \frac{\partial T_{s}}{\partial t}=\frac{\lambda_{1}}{t_{0}} \frac{\partial \psi_{s}}{\partial \tau} \\
& \frac{1}{r} \frac{\partial T_{s}}{\partial r}=\frac{\lambda_{1}}{r_{m}^{2}} \frac{1}{R} \frac{\partial \psi_{s}}{\partial R} \\
& \frac{\partial T_{s}}{\partial r}=\frac{\lambda_{1}}{r_{m}} \frac{\partial \psi_{s}}{\partial R} \\
& \frac{\partial^{2} T_{s}}{\partial r^{2}}=\frac{\lambda_{1}}{r_{m}^{2}} \frac{\partial^{2} \psi_{s}}{\partial R^{2}}
\end{aligned}
$$

where $r_{m}$ is the inside radius of the pipe containing the PCM. It is noted that for the phase change material, $\lambda_{1}$ and $\lambda_{2}$ are replaced by $\lambda_{1 s}$ and $\lambda_{2 s \text {. }}$

Substituting the dimensionless coefficients into the governing equation for the phase change material, simplifying and multiplying through by $r_{m}^{2} / \lambda_{1 s}$ to obtain

$$
\frac{\rho_{s}}{k_{s}} \frac{\Delta H_{s} r_{m}^{2}}{\lambda_{1 s} t_{0}} \frac{\partial \psi_{s}}{\partial \tau}=\frac{1}{R} \frac{\partial \psi_{s}}{\partial R}+\frac{\partial^{2} \psi_{s}}{\partial R^{2}}
$$

Now let

$$
\lambda_{1 s}=\lambda_{1} \frac{\lambda_{1 s}}{\lambda_{1}}=\lambda_{1} \beta_{s}
$$

Using the expressions for $\lambda_{1}$ (eqn 21) and $\lambda_{1 \mathrm{~s}}$ (eqn 22) from the temperature - enthalpy relationship, we have

$$
\begin{aligned}
& \beta_{s}=\frac{\Delta H_{s}}{c_{s}} \frac{c_{s}}{\Delta H_{s}}=1, \quad T_{s}<T_{1} \\
& \beta_{s}=\frac{2 \epsilon_{s}}{1} \frac{c_{s}}{\Delta H_{s}}=\frac{2 \epsilon_{s} c_{s}}{\Delta H_{s}}, \quad T_{1} \leq T_{s} \leq T_{2} \\
& \beta_{s}=\frac{\Delta H_{s}}{c_{s}} \frac{c_{s}}{\Delta H_{s}}=1, \quad T_{s}>T_{2}
\end{aligned}
$$

It is noted that for a phase change material with constant phase change temperature, $\mathrm{T}_{1}=\mathrm{T}_{2}=\mathrm{T}_{\mathrm{m}}$ $\epsilon_{s}=\left(T_{2}-T_{1}\right) / 2=0$ and hence $\beta_{s}=0$. Thus, $\beta_{s}$ is a phase change temperature range factor. Since $t_{0}$ can be taken quite arbitrary, we set $\frac{\rho_{s}}{k_{s}} \frac{\Delta H_{s} r_{m}^{2}}{\lambda_{1 s} t_{0}}=\frac{\rho_{s} \Delta H_{s} r_{m}^{2} c_{s}}{k_{s} \Delta H_{s} t_{0}}=\frac{\rho_{s} c_{s} r_{m}^{2}}{k_{s} t_{0}}=1$ to get $t_{0}=\frac{r_{m}^{2} \rho_{s} c_{s}}{k_{s}}$

Thus,

$$
t_{0}=\frac{r_{m}^{2} \rho_{s} c_{s}}{k_{s}}
$$

Substituting $\mathrm{t}_{0}$ and $\lambda_{1 s}$ into equation (34) and simplifying to obtain

$\frac{\rho_{s} \Delta H_{s} r_{m}^{2} k_{s} c_{s}}{k_{s} \beta_{s} \Delta H \rho_{s} r_{m}^{2} c_{s}} \frac{\partial \psi_{s}}{\partial \tau}=\frac{1}{R} \frac{\partial \psi_{s}}{\partial R}+\frac{\partial^{2} \psi_{s}}{\partial R^{2}}$

To give

$$
\frac{\partial \psi_{s}}{\partial \tau}=\beta_{s}\left\{\frac{1}{R} \frac{\partial \psi_{s}}{\partial R}+\frac{\partial^{2} \psi_{s}}{\partial R^{2}}\right\}
$$

The boundary conditions then become:

(i) $r=r_{m}, R=1$

We have $\quad-k_{s} \frac{\partial T_{s}}{\partial r}=u_{s}\left(T_{p 1}-T_{s}\right)$ from equation (3)

But

$$
\begin{aligned}
& -k_{s} \frac{\partial T_{s}}{\partial r}=-k_{s} \frac{\lambda_{1 s}}{r_{m}} \frac{\partial \psi_{s}}{\partial R} \\
& U_{s} T_{p 1}=U_{s} \lambda_{1} \psi_{p_{1}} \\
& U_{s} T_{s}=U_{s} \lambda_{1} \psi_{s} \\
& \therefore-k_{s} \frac{\lambda_{1 s}}{r_{m}} \frac{\partial \psi_{s}}{\partial R}=U_{s} \lambda_{1}\left(\psi_{p 1}-\psi_{s}\right)
\end{aligned}
$$


$\therefore-k_{s} \frac{\lambda_{1 s}}{r_{m}} \frac{\partial \psi_{s}}{\partial R}=U_{s} \lambda_{1} f\left(\psi_{p}, \psi_{s}\right)$

Where $f\left(\psi_{p 1}, \psi_{s}\right)=$ functions of

dimensionless enthalpy of plate 1 or temperature of plate 1 and dimensionless enthalpy of phase change material or temperature of phase change material.

Simplifying to obtain

$$
\begin{aligned}
& \frac{\partial \psi_{s}}{\partial R}=-\frac{U_{s} r_{m}}{k_{s}} \frac{\lambda_{1}}{\lambda_{1 s}} f\left(\psi_{p 1}, \psi_{s}\right) \\
& \frac{\partial \psi_{s}}{\partial R}=-\frac{U_{s} r_{m}}{k_{s}} \frac{1}{\beta_{s}} f\left(\psi_{p 1}, \psi_{s}\right) \\
& \therefore \frac{\partial \psi_{s}}{\partial R}=-\frac{S_{r_{1}}^{\prime}}{\beta_{s}}
\end{aligned}
$$

Discretizing equation (47) we have

$$
\frac{1}{4(\Delta R)}\left\{\begin{array}{l}
\psi_{s}(i+1, j+1)+\psi_{s}(i+1, j) \\
-\psi_{s}(i-1, j+1)-\psi_{s}(i-1, j)
\end{array}\right\}=-\frac{S_{r_{m}}^{\prime}}{\beta_{s}}
$$

Since $\mathrm{i}=\mathrm{m}$, we have

$\psi_{s}(m+1, j+1)+\psi_{s}(m+1, j)$

$=\psi_{s}(m-1, j+1)+\psi_{s}(m-1, j)-\frac{4(\Delta R) S_{r_{m}}^{\prime}}{\beta_{s}}$

where

$\psi_{p 1}$ is the plate dimensionless enthalpy and

$\psi_{s}$ is the phase change dimensionless enthalpy

(ii) Similarly, at $\mathrm{r}=0, \mathrm{R}=0$ and $\frac{\partial \psi}{\partial R}=0$

$\frac{\partial \psi_{s}}{\partial R}=0$

Discretizing equation (50) we obtain

$$
\frac{1}{4(\Delta R)}\left\{\begin{array}{l}
\psi_{s}(i+1, j+1)+\psi_{s}(i+1, j) \\
-\psi_{s}(i-1, j+1)-\psi_{s}(i-1, j)
\end{array}\right\}=0
$$

Since $\mathrm{i}=0$, we have

$$
\psi_{s}(1, j+1)+\psi_{s}(1, j)=\psi_{s}(-1, j+1)+\psi_{s}(-1, j)
$$

In order to achieve stable, accurate and fast solutions of the governing equations subject to the specified boundary conditions, CrankNicolson implicit finite difference scheme is utilized. The scheme, described in [9] and [10], has the advantage of being unconditionally stable, and has an accuracy of second order compared with the explicit method. The technique involves sweeping across time and space interval in one step. The resulting algebraic equations will therefore contain more than three unknowns at any given node in a time step. Consequently, the tridiagonal algorithm cannot be used alone to solve the equations. We therefore employ the Gauss-Seidel iterative scheme to solve the resulting equation in the tridiagonal algorithm using iterations.

For the numerical solution, the dimensionless forms of the partial differential coefficients are as follows:

$$
\begin{aligned}
& \frac{\partial \psi_{s}}{\partial \tau}=\frac{1}{\Delta \tau}\left\{\psi_{s}(i, j+1)-\psi_{s}(i, j)\right\} \\
& R=i(\Delta R) \\
& \frac{\partial \psi_{s}}{\partial R}=\frac{1}{4(\Delta R)}\left\{\begin{array}{l}
\psi_{s}(i+1, j+1)+\psi_{s} \\
(i+1, j)-\psi_{s}(i-1, j+1)-\psi_{s}(i-1, j)
\end{array}\right\} \\
& \frac{\partial^{2} \psi_{s}}{\partial R^{2}}=\frac{1}{2(\Delta R)^{2}}\left\{\begin{array}{l}
\psi_{s}(i+1, j+1)+\psi_{s}(i+1, j)-2 \psi_{s}(i, j+1) \\
-2 \psi_{s}(i, j)+\psi_{s}(i-1, j+1)+\psi_{s}(i-1, j)
\end{array}\right\}
\end{aligned}
$$

Substituting the finite difference form of the differential coefficient into the governing equation, we have that for any given pipe in the solar air heater, the finite difference forms of the equation (from equations $41,53,54,55$, and 56) become:

$$
\begin{aligned}
& \frac{\beta_{s}}{i(\Delta R)} \cdot \frac{1}{4(\Delta R)}\left\{\begin{array}{l}
\psi_{s}(i+1, j+1)+\psi_{s}(i+1, j) \\
-\psi_{s}(i-1, j+1)-\psi_{s}(i-1, j)
\end{array}\right\}+ \\
& \frac{\beta_{s}}{2(\Delta R)^{2}}\left\{\begin{array}{l}
\psi_{s}(i+1, j+1)+\psi_{s}(i+1, j)-2 \psi_{s}(i, j+1) \\
-2 \psi_{s}(i, j)+\psi_{s}(i-1, j+1)+\psi_{s}(i-1, j)
\end{array}\right\}= \\
& \frac{1}{\Delta \tau}\left\{\psi_{s}(i, j+1)-\psi_{s}(i, j)\right\}
\end{aligned}
$$

Collecting like terms and simplifying, they become

$$
\begin{aligned}
& \psi_{s}(i+1, j+1) \frac{\beta_{s}}{2(\Delta R)^{2}}\left\{\frac{1}{2 i}+1\right\}-\psi_{s}(i, j+1) \\
& \left\{\frac{1}{\Delta \tau}+\frac{\beta_{s}}{(\Delta R)^{2}}\right\}+\psi_{s}(i-1, j+1) \\
& \frac{\beta_{s}}{2(\Delta R)^{2}}\left\{1-\frac{1}{2 i}\right\}=-\psi_{s}(i+1, j) \frac{\beta_{s}}{2(\Delta R)^{2}} \\
& \left\{1+\frac{1}{2 i}\right\}+\psi_{s}(i, j)\left\{\frac{\beta_{s}}{(\Delta R)^{2}}-\frac{1}{\Delta \tau}\right\} \\
& +\psi_{s}(i-1, j) \frac{\beta_{s}}{2(\Delta R)^{2}}\left\{\frac{1}{2 i}-1\right\}
\end{aligned}
$$

This may be written compactly in the tridiagonal format as

$$
\begin{aligned}
& A_{1}(i, j) \psi_{s}(i+1, j+1)+B_{1}(i, j) \\
& \psi_{s}(i, j+1)+C_{1}(i, j) \psi_{s}(i-1, j+1)=D_{1}(i, j)
\end{aligned}
$$


where $A_{1}, B_{1}$, and $C_{1}$ have their significance.

The boundary conditions are

(i) At the outermost part of the pipe $r=r_{m}, i=m$; the finite difference formulation of the boundary condition from equation (49) is

$\frac{1}{4(\Delta R)}\left\{\begin{array}{l}\psi_{s}(i+1, j+1)+\psi_{s}(i+1, j) \\ -\psi_{s}(i-1, j+1)-\psi_{s}(i-1, j)\end{array}\right\}=-\frac{S_{r_{m}}^{\prime}}{\beta_{s}}$

Let $\mathrm{r}=\mathrm{r}_{\mathrm{m}}, \mathrm{i}=\mathrm{m}$, we have

$$
\frac{\beta_{s}}{4(\Delta R)}\left\{\begin{array}{c}
\psi_{s}(m+1, j+1)+\psi_{s}(m+1, j) \\
-\psi_{s}(m-1, j+1)-\psi_{s}(m-1, j)
\end{array}\right\}=-S_{r_{m}^{\prime}}^{\prime}
$$

From equation (52) we have

$\psi_{s}(m+1, j+1)+\psi_{s}(m+1, j)=$

$\psi_{s}(m-1, j+1)+\psi_{s}(m-1, j)-\frac{4(\Delta R) S_{r_{m}}^{\prime}}{\beta_{s}}$

Using the general node equation, equation (61), substituting $\mathrm{i}=\mathrm{m}$ and simplifying further we have

$\left[\psi_{s}(m+1, j+1)+\psi_{s}(m+1, j)\right] \frac{\beta_{s}}{2(\Delta R)^{2}}\left\{1+\frac{1}{2 m}\right\}-\psi_{s}(m, j+1)$

$\left\{\frac{1}{\Delta \tau}+\frac{\beta_{s}}{(\Delta R)^{2}}\right\}+\psi_{s}(m-1, j+1)$

$\frac{\beta_{s}}{(\Delta R)^{2}}\left\{1-\frac{1}{2 m}\right\}=\psi_{s}(m, j)\left\{\frac{\beta_{s}}{(\Delta R)^{2}}-\frac{1}{\Delta \tau}\right\}$

$+\psi_{s}(m-1, j) \frac{\beta_{s}}{2(\Delta R)^{2}}\left\{\frac{1}{2 m}-1\right\}$

Substituting equation (49) into equation (61) and after rearranging to get

$\psi_{s}(m-1, j+1) \frac{\beta_{s}}{2(\Delta R)^{2}}\left\{\left(1+\frac{1}{2 m}\right)+\left(1-\frac{1}{2 m}\right)\right\}$

$+\psi_{s}(m-1, j) \frac{\beta_{s}}{2(\Delta R)^{2}}\left\{\left(1+\frac{1}{2 m}\right)-\left(1-\frac{1}{2 m}\right)\right\}$

- $\psi_{s}(m, j+1)\left\{\frac{1}{\Delta \tau}+\frac{\beta_{s}}{(\Delta R)^{2}}\right\}=\psi_{s}(m, j)\left\{\frac{\beta_{s}}{(\Delta R)^{2}}-\frac{1}{\Delta \tau}\right\}+\frac{2 S_{r_{m}}^{\prime}}{(\Delta R)}$

Solving and simplifying we have

$\psi_{s}(m-1, j+1) \frac{\beta_{s}}{(\Delta R)^{2}}-\psi_{s}(m, j+1)\left\{\frac{1}{\Delta \tau}+\frac{\beta_{s}}{(\Delta R)^{2}}\right\}=$

$-\psi_{s}(m-1, j) \frac{\beta_{s}}{2 m(\Delta R)^{2}}+\psi_{s}(m, j)\left\{\frac{\beta_{s}}{(\Delta R)^{2}}-\frac{1}{\Delta \tau}\right\}+\frac{2 S_{r_{m}}^{\prime}}{\Delta R}$

In tridiagonal terms, this may be rewritten as $A(m, j) \psi_{s}(m-1, j+1)+B(m, j) \psi_{s}(m, j+1)=D(m, j$

where $A(m, j), B(m, j)$,and $D(m, j)$ have their significance

Similarly, at the centre of the pipe, $r=0$, the finite difference formulation of the boundary condition from equation (51) becomes

$$
\begin{aligned}
& \frac{1}{4(\Delta R)}\left\{\begin{array}{l}
\psi_{s}(i+1, j+1)+\psi_{s}(i+1, j) \\
-\psi_{s}(i-1, j+1)-\psi_{s}(i-1, j)
\end{array}\right\}=0 \\
& \therefore \psi_{s}(i+1, j+1)+\psi_{s}(i+1, j) \\
& -\psi_{s}(i-1, j+1)+\psi_{s}(i-1, j)=0 \\
& \text { For } r=0 \quad \therefore i=0
\end{aligned}
$$

Hence

$$
\psi_{s}(i, j+1)+\psi_{s}(1, j)-\psi_{s}(-1, j+1)-\psi_{s}(-1, j)=0
$$

From equation (52), we have

$$
\begin{aligned}
& \psi_{s}(1, j+1)+\psi_{s}(1, j)=\psi_{s}(-1, j+1)+\psi_{s}(-1, j) \\
& \Rightarrow \psi_{s}(-1, j+1)+\psi_{s}(-1, j)=\psi_{s}(1, j+1)+\psi_{s}(1, j)
\end{aligned}
$$

From equation (58), the general node equation, and substituting $\mathrm{i}=0$, we obtain

$\psi_{s}(1, j+1) \frac{\beta_{s}}{2(\Delta R)^{2}}-\psi_{s}(0, j+1)\left\{\frac{1}{\Delta \tau}+\frac{\beta_{s}}{(\Delta R)^{2}}\right\}+\psi_{s}(-1, j+1)$

$\frac{\beta_{s}}{2(\Delta R)^{2}}=-\psi_{s}(1, j) \frac{\beta_{s}}{2(\Delta R)^{2}}$

$+\psi_{s}(0, j)\left\{\frac{\beta_{s}}{(\Delta R)^{2}}-\frac{1}{\Delta \tau}\right\}-\psi_{s}(-1, j) \frac{\beta_{s}}{2(\Delta R)^{2}}$

Rearranging and substituting equation (67) into equation (68), we have

$\psi_{s}(1, j+1) \frac{\beta_{s}}{2(\Delta R)^{2}}-\psi_{s}(0, j+1)$

$\left\{\frac{1}{\Delta \tau}+\frac{\beta_{s}}{(\Delta R)^{2}}\right\}+\left\{\psi_{s}(1, j+1)+\psi_{s}(1, j)\right\} \frac{\beta_{s}}{2(\Delta R)^{2}}=$

- $\psi_{s}(1, j) \frac{\beta_{s}}{2(\Delta R)^{2}}+\psi_{s}(0, j)\left\{\frac{\beta_{s}}{(\Delta R)^{2}}-\frac{1}{\Delta \tau}\right\}$

Solving and simplifying, we have

$\psi_{s}(1, j+1)\left\{\frac{\beta_{s}}{2(\Delta R)^{2}}+\frac{\beta_{s}}{2(\Delta R)^{2}}\right\}-\psi_{s}(0, j+1)\left\{\frac{1}{\Delta \tau}+\frac{\beta_{s}}{(\Delta R)^{2}}\right\}=$

$-\psi_{s}(1, j)\left\{\frac{\beta_{s}}{2(\Delta R)^{2}}+\frac{\beta_{s}}{2(\Delta R)^{2}}\right\}+\psi_{s}(0, j)\left\{\frac{\beta_{s}}{(\Delta R)^{2}}-\frac{1}{\Delta \tau}\right\}$

In tridiagonal terms, this may be rewritten as

$B(0, j) \psi_{s}(1, j+1)+C(m, j)(0, j+1)=D(0, j)$

where $B(0, j), C(0, j)$, and $D(0, j)$ has their significance.

We assume one-dimensional heat transfer in cylindrical coordinates and constant thermal conductivity. The number of nodes is specified to be $m=7$, and they are chosen to be at the half diameter of the tube, one at the thermal symmetry of the pipe and one at the edge of the pipe as shown in figure 3 . The nodal spacing $\Delta r=\frac{R}{m-1}$ 


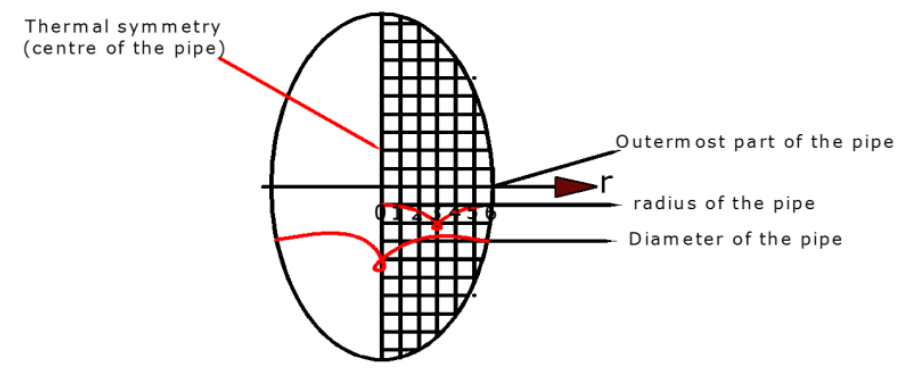

Figure 3: The Nodal Points

We number the nodes $0,1,2,3,4,5$ and 6 . The temperature at node 0 is given to be $\mathrm{T}_{0}$, and the temperatures at nodes $1,2,3,4,5$, and 6 are to be determined. This problem involves only six unknown nodal temperatures, and thus we need to have only six equations to determine them uniquely. These equations are obtained by applying crank-Nicolson implicit finite difference methods to nodes $1,2,3,4,5$, and 6 .

Nodes 1, 2, 3, 4, and 5 are interior nodes and the finite difference formulation at those nodes are obtained from the governing equation (the parabolic equation) and is given by equation 58 as

$$
\begin{aligned}
& \psi_{s}(i+1, j+1) \frac{\beta_{s}}{2(\Delta R)^{2}}\left\{\frac{1}{2 i}+1\right\}-\psi_{s}(i, j+1)\left\{\frac{1}{\Delta \tau}+\frac{\beta_{s}}{(\Delta R)^{2}}\right\} \\
& +\psi_{s}(i-1, j+1) \frac{\beta_{s}}{2(\Delta R)^{2}}\left\{1-\frac{1}{2 i}\right\}= \\
& -\psi_{s}(i+1, j) \frac{\beta_{s}}{2(\Delta R)^{2}}\left\{1+\frac{1}{2 i}\right\}+\psi_{s}(i, j) \\
& \left\{\frac{\beta_{s}}{(\Delta R)^{2}}-\frac{1}{\Delta \tau}\right\}+\psi_{s}(i-1, j) \frac{\beta_{s}}{2(\Delta R)^{2}}\left\{\frac{1}{2 i}-1\right\}
\end{aligned}
$$

Node 6 is a boundary node. It is at the edge of pipe. The finite difference formulation at that node is obtained by taking the boundary condition at that node which is given by equation 63 as

$$
\begin{aligned}
& \psi_{s}(m-1, j+1) \frac{\beta_{s}}{(\Delta R)^{2}}-\psi_{s}(m, j+1)\left\{\frac{1}{\Delta \tau}+\frac{\beta_{s}}{(\Delta R)^{2}}\right\}= \\
& -\psi_{s}(m-1, j) \frac{\beta_{s}}{2 m(\Delta R)^{2}}+\psi_{s}(m, j)\left\{\frac{\beta_{s}}{(\Delta R)^{2}}-\frac{1}{\Delta \tau}\right\}+\frac{2 S_{r_{m}}^{\prime}}{\Delta R}
\end{aligned}
$$

Node 0 is a boundary node. It is the centre of the circular pipe which is at the thermal symmetry. And the finite difference formulation at that node is obtained by taking the boundary condition at that node which is given by equation 70 as

$$
\begin{aligned}
& \psi_{s}(1, j+1)\left\{\frac{\beta_{s}}{2(\Delta R)^{2}}+\frac{\beta_{s}}{2(\Delta R)^{2}}\right\}-\psi_{s}(0, j+1)\left\{\frac{1}{\Delta \tau}+\frac{\beta_{s}}{(\Delta R)^{2}}\right\} \\
& =-\psi_{s}(1, j)\left\{\frac{\beta_{s}}{2(\Delta R)^{2}}+\frac{\beta_{s}}{2(\Delta R)^{2}}\right\}+\psi_{s}(0, j)\left\{\frac{\beta_{s}}{(\Delta R)^{2}}-\frac{1}{\Delta \tau}\right\}
\end{aligned}
$$

Nodes 1, 2, 3, 4, 5 and 6 form a system of six equations in six unknowns $\Psi_{15}, \Psi_{25}, \Psi_{35}, \Psi_{45}, \Psi_{55}$, and $\Psi_{65}$. Substituting the given quantities and simplifying gave the system of equations.

\section{Results and Discussions}

The discrete forms of the governing equations modeling the thermal behaviour of the solar air heater were solved numerically. An existing computer programme in BASIC known as EGGINC reported in [2] is adapted to implement the numerical solution of the equations and produce performance results. The programme adapted by [2] and modified by [12] to suit his analysis in radial tubes is a sequential modular programme consisting the main programme and some other subprogrammes. The major subgrogramme of the system is named PCM to implement the numerical solutions of the equations. This programme models the air heater one dimensional radial heat conduction in a phase change material (PCM) heat storage system encapsulated in cylindrical pipes. Other subprogrammes read and analyze air heater chamber, absorber plate and fins, solar radiation data and compute the particular amount absorbed at any given time. A number of the subroutines calculated heat transfer coefficients for natural or forced convection under various conditions, while a group of other subroutines calculate the thermo physical properties of air at any required temperature. Subprogrammes used in this work were also available to implement standard numerical procedures such as function integration using Simpson's rule and the solution of tridiagonal algebraic equations.

The data for the experimental and predicted values were reported in [12] for $12^{\text {th }}, 13$ and $14^{\text {th }}$ June, 2006, as shown in figs (i) to (iii),the ambient temperatures were the initial conditions for each day. The collector temperature starts from the initial condition and rises to its maximum value near solar noon corresponding to temperatures of $60^{\circ} \mathrm{C}$ to $70^{\circ} \mathrm{C}$. It begins to decrease thereafter as intensity falls. It was observed that these happened in all cases as shown the figures. The predicted temperatures were plotted alongside the experimental values in figures (i) to (iii). In Figure 4 that was June $12^{\text {th }}$, 2006 , the predicted value was about $76^{\circ} \mathrm{C}$ and the experimental value was about $70^{\circ} \mathrm{C}$. They were close to within $6^{\circ} \mathrm{C}$. On June 13,2006 , the predicted value was $76^{\circ} \mathrm{C}$ and the experimental value was about $56^{\circ} \mathrm{C}$. They were close to within $20^{\circ}$ C.Lastly, on the June 14,2006 , the predicted 
value was about $74^{\circ} \mathrm{C}$ and the experimental value was about $68^{\circ} \mathrm{C}$. They were close to within $4^{\circ} \mathrm{C}$. The values shown above is at the upper limit as shown in the graphs.

At the lower limit in the discussion below the predicted value was $40^{\circ} \mathrm{C}$ and the experimental value was $22^{\circ} \mathrm{C}$. They were close to within $18^{\circ} \mathrm{C}$. That was on June 12,2006 . On the $13^{\text {th }}$ June, 2006, the predicted value was $40^{\circ} \mathrm{C}$ and the experimental value was $23^{\circ} \mathrm{C}$. They were close to within $17^{\circ} \mathrm{C}$. Lastly, on the $14^{\text {th }}$ June, 2006 , the predicted value was about $38^{\circ} \mathrm{C}$ and the experimental value was $25^{\circ} \mathrm{C}$. They were close to within $13^{\circ} \mathrm{C}$.The system operates over the ambient temperature range of $18^{\circ} \mathrm{C}-38^{\circ} \mathrm{C}$ and daily global irradiation range of $4.9-20.1 \mathrm{MJ} / \mathrm{m}^{2}$ daily. The cumulative useful efficiency was about $13.3 \%$. The reason for the low efficiency was as a result of gloomy and wet days in June. It is observed that from Figures4 - 6, the predicted curve was close enough to the experimental curve within the lower limits of about $6.5^{\circ} \mathrm{C}$ and upper limits of about $13^{\circ} \mathrm{C}$.

The predicted values on the system (the solar air heater) for 3 different days in June at Nsukka, Nigeria (latitude $7^{0} \mathrm{~N}$ ) of which the initial condition (the ambient temperature and irradiance) for each day are taken from experimental data. The night time performance was not tested. The daily global irradiation covered the range $4.9-20.1 \mathrm{MJ} / \mathrm{m}^{2}$-day, while the ambient temperature over the period varied within the range $18.5-36.0^{\circ} \mathrm{C}$. The initial conditions for each day are taken from the experimental data.

Below are the graphs of Figures $4-6$ comparing the experimental temperatures and the predicted temperatures, also shown in the graphs are the irradiance and ambient temperatures.

\section{Conclusion}

The finite difference method presented in this work has been employed to deal with onedimensional heat conduction problems analyzed in cylindrical coordinate. The computer procedure has been found to be reasonable within the trend or within acceptable limits. In this work we considered the phase change material resulting from phase transformations, the melting/freezing problem. Numerical solution of this problem using Crank-Nicolson implicit finite difference scheme is obtained. The onedimensional radial heat conduction of a phase change materials (PCM) heat storage system encapsulated in cylinders housed in a single glazed flat plate natural circulation solar air heater is presented. Theoretical modeling of the solar air heater predicted the temperature values as compared to the experimental values which agree closely with experimental data.

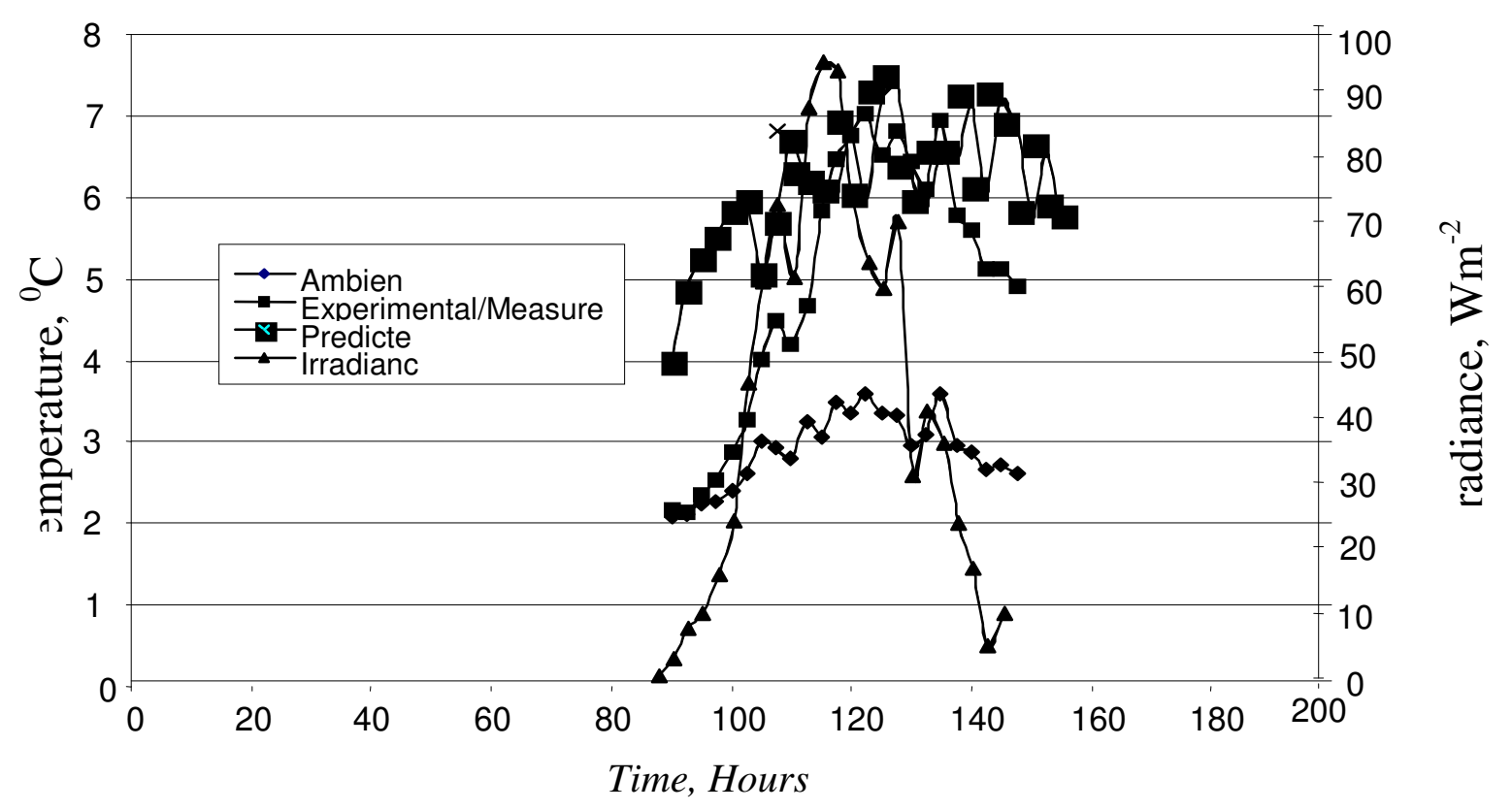

Figure 4: Comparison between the measured and predicted absorber plate temperatures on June 12 
Natural Circulation Solar Air Heater With Phase Change Material Energy Storage, A. I. Obi, et al

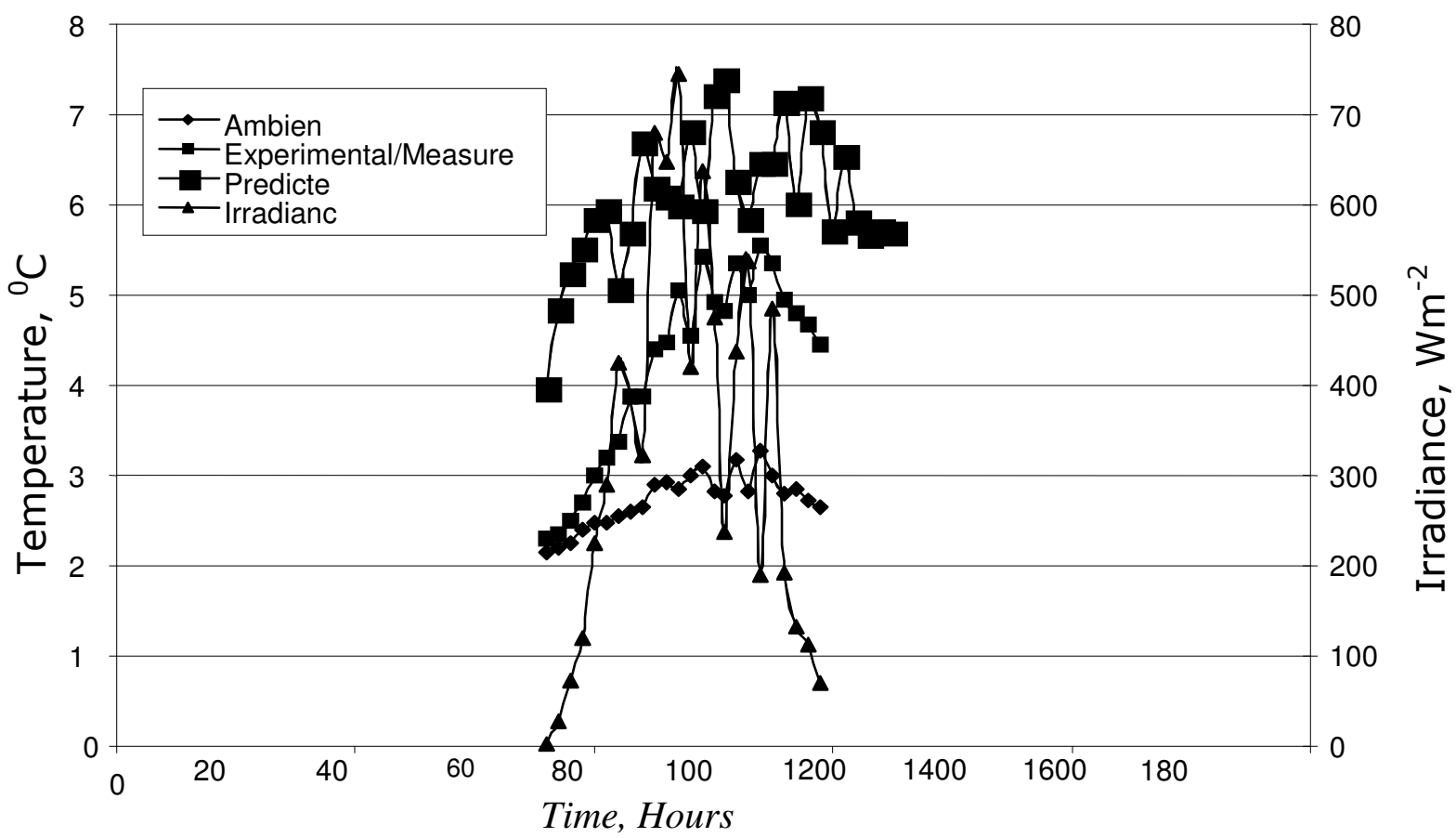

Figure 5: Comparison between the measured and predicted absorber plate temperatures on June 13

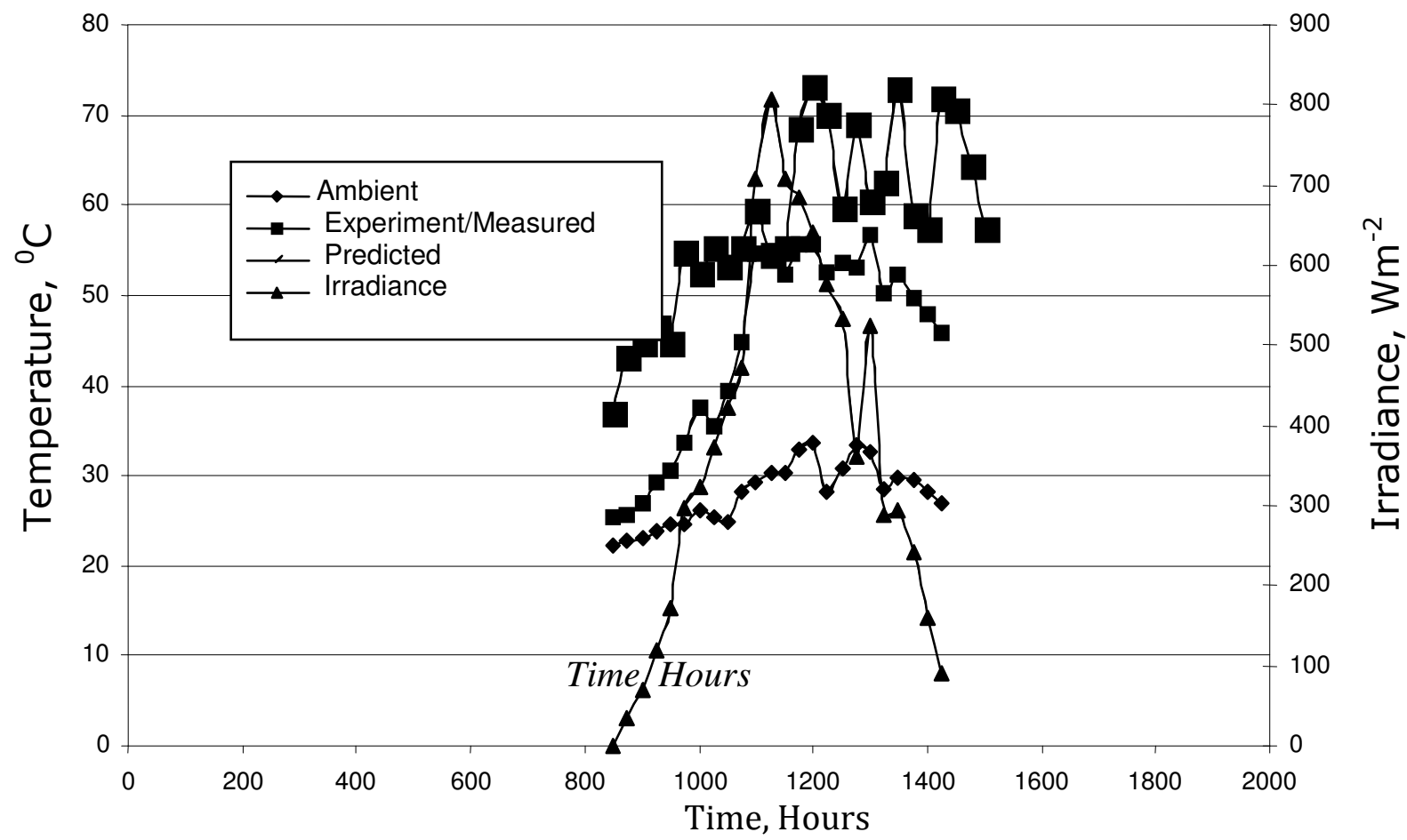

Figure 6: Comparison between the measured and predicted absorber plate temperatures on June 13

\section{References}

[1] Voller, V. and Cross, .M. (1981). Accurate Solutions of Moving Boundary Problems Using the Enthalpy Method. Int. J. Heat Mass Transfer 24, $545-556$.

[2] Enibe, S.O. (2003). Thermal Analysis of a Natural Circulation Solar Air Heater With Phase Change Material Energy Storage. Renewable Energy 28 (2003) :2269-2299.
[3] Rabin Y., Bar-Niv I. and Mike B. (1995) Integrated Solar Collector Storage System Based on a Salthydrate Phase Change Material. Solar Energy 55 (6): 435-444

[4] Macedo, I. C. and Altemani, C.A.C. Experimental Evaluation of Natural Convection Solar Air Heaters. Solar Energy 20, 367 - 369.

[5] Ekechukwu, O.V. and Norton, B. (1999). Review of Solar Energy Drying Systems (2): An Overview of 
Solar Drying Technology. Energy Convers. Mgmt 40, 614-655.

[6] Diamante, L. M. and Munro, P.A. (1993). Mathematical Modeling of the Thin Layer Drying of Sweet Potato Slices. Solar Energy 51 (4) : 271276.

[7] Fohr, J.P and Figueiredo, A.R. (1980). Agricultural Solar Air Collectors: Design and Performances.

[8] Palaniappan, C. and Subramanian, S.V. (1998). Economics of Solar Air Pre- heating in South Indian Tea Factories: A Case Study. Solar Energy 63 ( 1 ): 31- 37.

[9] Erwin Kreyszig (2006). Advanced Engineering Mathematics. $8^{\text {th }}$ edition, New York: John Wiley and Sons, Inc.

[10] Carnahan B., H.A. Luther and James O. Wilkes (1969). Applied Numerical Methods. New York: John Wiley and Sons Inc.

[11] Frank P. Incropera and David P. Dewitt (1996). Introduction to Heat Transfer, $3^{\text {rd }}$ ed., New York: John Wiley and Sons Inc.

[12] Obi A.I., 2008, "Performance Simulation of Natural Circulation Solar Air Heating System with Phase Change Material Energy Storage for Low Temperature Applications" M.Eng. Project, University of Nigeria, Nsukka.

[13] Duffie, J. A. and Beckman, W. A. (1991). Solar Engineering of Thermal Processes, $2^{\text {nd }}$ ed., New York: John Wiley and Sons.

\section{Nomenclature}

A First term in tridiagonal equation

B Second term in tridiagonal equation

C Third term in tridiagonal equation

$c_{s} \quad$ Specific heat of the phase change material

D Tube or pipe diameter

Last term in tridiagonal equation.

G Solar radiation intensity, $\mathrm{Wm}^{-2}$

h Enthalpy, kJ kg-1

$\mathrm{H}$ Total enthalpy, $\mathrm{kJ} \mathrm{kg}^{-1}$

i Node identifier in r-direction j Node identifier in time direction

$\mathrm{k} \quad$ Thermal conductivity, $\mathrm{Wm}^{-1} \mathrm{k}^{-1}$

$\mathrm{m} \quad$ Number of nodes in $\eta$ direction in a pipe containing PCM

$r \quad$ Radial distance, $m$

$r_{o} \quad$ Centre of the pipe containing the PCM, $m$

$r_{m} \quad$ Radius at the edge or inside radius of the pipe containing the PCM, $\mathrm{m}$

$\mathrm{R}$ Dimensionless distance in the radial direction i.e. r-direction

$\mathrm{S}$ Absorbed radiation, $\mathrm{Wm}^{-2}$

$\mathrm{S}^{\prime} \quad$ Dimensionless net heat flux

$\mathrm{T}$ Temperature, $\mathrm{K}$

$\mathrm{T}_{\mathrm{f}} \quad$ Fin or plate temperature, $\mathrm{K}$

$\mathrm{t}$ Time, $\mathrm{s}$

$\mathrm{t}_{\mathrm{o}} \quad$ Arbitrary time, $\mathrm{s}$

$\mathrm{U} \quad$ Heat transfer coefficient, $\mathrm{Wm}^{-2} \mathrm{~K}^{-1}$

\section{Greek Letters}

$\alpha \quad$ Thermal diffusivity, $\mathrm{m}^{2} \mathrm{~s}^{-1}$

$\beta_{\mathrm{s}} \quad$ PCM phase change temperature range factor defined in equations $46-50$.

$\delta \quad$ Thickness, $\mathrm{m}$

$\varepsilon \quad$ Implicit procedure.

$\theta \quad$ Temperature

Tangential direction to the pipe containing the PCM (angular), $r$

$\varepsilon_{s} \quad$ Half the difference between upper and lower melting temperature of the PCM, K.

$\lambda$ Parameter defined in equation 3-49

$\lambda_{1} \quad$ Parameter defined in equation $4-24$

$\lambda_{2} \quad$ Parameter defined in equation $4-24$

$\psi \quad$ Dimensionless enthalpy

$\tau \quad$ Dimensionless time

$\Delta \quad$ Change in quantity

$\phi \quad$ Temperature - irradiance function defined in equation.

$\delta \quad$ Partial change or partial derivative

\section{Subscripts}

a Ambient, absorbed

$\mathrm{s} \quad$ Phase change material 